\title{
Distribución y viabilidad de cisticercos de Taenia saginata en los cortes de carne de la canal de bovinos naturalmente infectados $\#$
}

\author{
Distribution and viability of cyticerci of Taenia saginata in meat cuts from naturally infected beef \\ F Cayo $^{\text {ab, }}$ G Valenzuela , E Paredes ${ }^{\mathrm{c}}$, V Ruíz ${ }^{\mathrm{d}}$, C Gallo ${ }^{\mathrm{b}}$ \\ ${ }^{a}$ Becario AGCI, Programa de Magíster en Ciencias mención Salud Animal, Facultad de Ciencias Veterinarias, \\ Universidad Austral de Chile, Valdivia, Chile. \\ ${ }^{\mathrm{b}}$ Instituto de Ciencia Animal, Facultad de Ciencias Veterinarias, Universidad Austral de Chile, Valdivia Chile. \\ ${ }^{\mathrm{c}}$ Instituto de Patología Animal, Universidad Austral de Chile, Valdivia, Chile. \\ d Actividad Privada y Corporación de la Carne, Osorno, Chile
}

\begin{abstract}
SUMMARY
The anatomical distribution and viability of cysticerci of Taenia saginata in naturally infected cattle slaughtered in southern Chile was determined. The cattle were inspected post mortem according to official chilean procedures, where mandatory inspection sites are: heart, tongue, masseter, oesophagus, diaphragm and superficial observation of the carcass. Twenty carcasses diagnosed as positive during inspection were chosen at random and their left half sides were separated into the 33 official meat cuts; these were completely dissected into approximately $0.5 \mathrm{~cm}$ thick slices and number of cysts determined by visual inspection. Lesions compatible with cysticerci of Taenia saginata were diagnosed according to official procedures in 148 cattle ( $0.5 \%$ prevalence) at mandatory sites, finding that $64.9 \%$ of them were located in heart, $33.8 \%$ in masseter muscles, $0.7 \%$ in oesophagus and $0.7 \%$ in the carcasses. At histological examination, $82.9 \%$ of the lesions were diagnosed as nonviable cysts and only $5.1 \%$ were viable; $10.7 \%$ corresponded to other diagnoses (mostly myositis and myocarditis, followed by schwannoma in heart and actynomycosis in tongue) and in $1.3 \%$ no alteration could be found. Fourteen cysts were found in the meat cuts of the 20 positive to cysticerci of Taenia saginata in carcasses, all were nonviable; cysts were found in tenderloin, point end brisket, rump heart, silverside, cube roll cover, ribs, topside, flanck meat, bolar and thin skirt. It is concluded that chances of finding cysticerci in meat cuts of cattle diagnosed positive to cysticerci of Taenia saginata at slaughter inspection is limited, especially when animals are infected in a mild form, as it happens in Chile.
\end{abstract}

Key words: Taenia saginata, cysticerci, anatomical distribution, viability.

\section{RESUMEN}

Se determinó la distribución anatómica y viabilidad de los cisticercos de Taenia saginata en bovinos naturalmente infectados, faenados en el sur de Chile. Los animales fueron inspeccionados post mórtem según los procedimientos chilenos oficiales en los puntos obligatorios: corazón, lengua, maseteros, esófago, diafragma y superficie de canal. Se tomaron al azar 20 canales de bovinos diagnosticados positivos a la inspección, y sus mitades izquierdas fueron despostadas en los 33 cortes oficiales; éstos fueron completamente disecados en láminas de aproximadamente $0,5 \mathrm{~cm}$ de grosor, contabilizando el número de cisticercos encontrados por inspección visual en cada corte. Lesiones compatibles con cisticercos de Taenia saginata se encontraron a la inspección oficial en 148 bovinos ( $0,5 \%$ de prevalencia), encontrando 64,9\% en corazón, 33,8\% en músculos maseteros, $0,7 \%$ en esófago y $0,7 \%$ en la canal. Al examen histológico de las lesiones, se encontró que 82,9\% eran cisticercos no viables (degenerados) y sólo 5,1\% eran viables; $10,7 \%$ correspondieron a otros diagnósticos (principalmente miositis, seguido de miocarditis en corazón y actinobacilosis en lengua) y en $1,3 \%$ de los casos no se encontraron alteraciones. Se encontraron 14 lesiones compatibles con cisticercosis en las 20 canales positivas a cisticercos de Taenia saginata a la inspección; todas fueron confirmadas positivas al análisis histológico y todas eran quistes degenerados. Estos cisticercos estaban distribuidos en filete, tapapecho, asiento, ganso, plateada, asado de tira, posta negra, tapabarriga, posta de paleta y entraña. Se concluye que la posibilidad de encontrar cisticercos en cortes de carne es limitada, especialmente en animales infectados en forma leve, como sucede en Chile.

Palabras clave: Taenia saginata, cisticercos, distribución anatómica, viabilidad.

\section{INTRODUCCIÓN}

La cisticercosis bovina es una enfermedad que afecta al músculo estriado del ganado y es causada por el cisticerco (forma larval) de Taenia saginata, cuyo esta-

Aceptado: 10.01.2013

\# Financiado por el proyecto INNOVA-CORFO 07CN13IAT247

* cgallo@uach.cl do adulto se localiza en el intestino delgado del hombre (Wanzala y col $2003^{\text {b }}$ ). La carne cruda o insuficientemente cocinada, infectada con cisticercos viables, es fuente de infección para los humanos, mientras que los bovinos se infectan al consumir pasto o agua contaminada con huevos de T. saginata (Martano y col 2010, Ogunremi y Benjamin 2010, Eichenberger y col 2011).

La cisticercosis tiene importancia como problema de salud pública al ser una zoonosis y tener interés socioe- 
conómico debido a las pérdidas que produce a nivel de mataderos (OPS/OMS 2003, Regassa y col 2009). Desde el punto de vista de la producción de carne, la cisticercosis bovina produce serias pérdidas económicas en la industria del ganado (Yoder y col 1994, Giesecke 1997), estimándose una reducción de entre 30 a $45 \%$ del valor de una canal afectada (Geerts 1990). En Chile, según la inspección oficial canales con infecciones leves (hasta cuatro cisticercos según MINSAL 2002), son aptas para consumo humano previo saneamiento; las pérdidas derivan de la necesidad de congelar la carne como medida de saneamiento, la consiguiente devaluación económica del producto y el decomiso de subproductos comestibles. En el caso de las canales decomisadas (más de 4 cisticercos) la pérdida es prácticamente total (MINSAL 2002) o simplemente son incineradas. Por tanto es importante dar debida atención a esta enfermedad para mejorar la calidad y cantidad de carne para satisfacer el consumo local e incrementar los ingresos por las exportaciones (Kebede 2008).

Los cisticercos a menudo pueden ser detectados durante la inspección rutinaria de carnes en los sitios oficiales de inspección como el corazón, músculos maseteros internos y externos, músculos pterigoideos, lengua, esófago, diafragma y observación superficial de la canal (MINSAL 2002, Abunna y col 2008, Kebede y col 2009, Scandrett y col 2009). Sin embargo Minozzo y col (2002) han demostrado una amplia distribución del metacestodo de la T. saginata en los músculos del bovino. De acuerdo a numerosos estudios de infecciones naturales y experimentales en el ganado, es discutible si el parásito tiene verdaderamente sitios de predilección o puede estar distribuido en cualquier sitio (Kyvsgaard y col 1990, Maeda y col 1996, OPS/OMS 2003). Según Wanzala y col (2003 $\left.{ }^{\mathrm{a}, \mathrm{b}}\right)$ la mayoría de los cisticercos se encuentra en músculos esqueléticos (24-74\%). Por lo anterior, es importante determinar la distribución de los cisticercos a nivel del tejido muscular esquelético de bovinos naturalmente infectados, ya que estos tejidos son destinados a consumo humano posterior al saneamiento. El objetivo de esta investigación fue determinar la distribución y viabilidad de cisticercos de Taenia saginata en los sitios de inspección oficial y en los cortes de carne en canales de bovinos naturalmente infectados.

\section{MATERIAL Y MÉTODOS}

\section{DISTRIBUCIÓN ANATÓMICA DE LOS CISTICERCOS}

Se utilizaron los registros de la inspección oficial de la canal y vísceras, realizada por los médicos veterinarios oficiales del Servicio Agrícola y Ganadero (SAG) de acuerdo a la Norma General Técnica N ${ }^{\circ} 62$ (MINSAL 2002), en el periodo de junio a diciembre de 2010 en una planta faenadora. Esta norma establece la inspección visual junto con la palpación e incisión como métodos oficiales de diagnóstico de la cisticercosis bovina, efec- tuada en los puntos de inspección obligatorios: corazón, lengua, maseteros, esófago, diafragma y la superficie de la canal expuesta durante el procedimiento de inspección (MINSAL 2002). De la misma planta se obtuvieron al azar 20 medias canales de bovino diagnosticadas como positivas a cisticercosis, correspondientes a infección leve (19) y masiva (1), según la inspección oficial. Posterior a la inspección de estas canales y previo al saneamiento o decomiso según el caso, se realizó el desposte total de las medias canales izquierdas, según la norma chilena oficial de cortes de carne de bovino (INN 1999). Cada uno de los 33 cortes oficiales fue seccionado en láminas de aproximadamente $0,5 \mathrm{~cm}$ de grosor mediante cuchillos, usando la técnica de Scandrett y col (2009).

Cada lámina de carne se examinó por ambos lados para visualizar lesiones compatibles con cisticercosis (quistes) en un ambiente bien iluminado, registrándolas según número y corte en que se ubicaban (Scandrett y col 2009). Todos los quistes encontrados fueron identificados individualmente, fijados en formol al $10 \%$ y enviados a los Laboratorios de Parasitología y Anatomía Patológica Veterinaria de la Universidad Austral de Chile, para su análisis macroscópico e histopatológico. Adicionalmente, fueron remitidos al Laboratorio todos los quistes encontrados a la inspección oficial en el periodo de estudio, indicando de qué órgano o sitio de inspección procedían.

\section{DETERMINACIÓN DE LA VIABILIDAD DE LOS CISTICERCOS}

Se realizó el examen macroscópico de todos los quistes para determinar si éstos eran viables o degenerados. Los quistes suaves al tacto y llenos de líquido, con escólex visible, fueron clasificados como viables; el diagnóstico estuvo basado en la ausencia de ganchos y rostelo en el escólex mediante un examen con microscopio estereoscópico (OIE 2009, Scandrett y col 2009). Los cisticercos duros al tacto y al corte, que contenían material caseoso o cremoso, de color verdoso o amarillento fueron clasificados como cisticercos degenerados (Ogunremi y col 2004).

Con los antecedentes obtenidos se determinó si se estaba frente a un cisticerco viable; en caso contrario, se procedió a la realización de un examen histopatológico. Para ello los metacestodos incluidos junto con el tejido muscular fueron fijados en formalina y su estudio histológico fue evaluándolos mediante cortes seriados (5-6 $\mu \mathrm{m}$ de grosor), que fueron procesados por la técnica habitual para inclusión en parafina y coloración con hematoxilina-eosina descrito por (Luna 1968). La identificación de cisticercos degenerados se fundamentó en el hallazgo de los corpúsculos calcáreos, concreciones concéntricas de sales que tienen un tamaño aproximado de 5-10 $\mu \mathrm{m}$, en el parénquima del parásito (OIE 2009). Con los datos obtenidos se realizó un análisis estadístico descriptivo. 


\section{RESULTADOS Y DISCUSIÓN}

Durante el período en estudio se registraron 148 casos de cisticercosis, que representaron un $0,5 \%$ de prevalencia, similar a la registrada por Cayo (2011) quien reportó un $0,6 \%$ en un estudio realizado en tres plantas faenadoras de carnes del sur de Chile en la gestión 2010. De los casos registrados, $132(89,2 \%)$ presentaron a la inspección oficial un solo quiste, $13(8,8 \%)$ presentaron dos quistes y 2 $(1,3 \%)$ presentaron tres quistes, totalizando un $99,3 \%$ con infección leve; un solo caso de los 148 (0,7\%) correspondió a infección masiva.

La distribución anatómica de los cisticercos en los casos identificados en la inspección oficial de canales mostró que la localización más frecuente correspondió al corazón (96 quistes), músculo masétero (50 quistes), esófago (1 quiste) y canal (1 quiste). Según Carvalho y col (2006) los cisticercos se encuentran con mayor frecuencia en los músculos más irrigados, en particular en el corazón y músculos masticatorios, lo que concuerda con los resultados de nuestro estudio donde el $98,6 \%$ de los hallazgos a la inspección oficial ocurrió en estos lugares. La ubicación preferencial de los cisticercos en el corazón podría atribuirse a la pronta respuesta inflamatoria del músculo cardiaco y debido a que la reabsorción de los cisticercos degenerados en este sitio se produce más lentamente (Carvalho y col 2006, Scandrett y col
2009). También Fernandes y Buzetti (2001) observaron un $97,5 \%$ de los casos de cisticercosis del ganado en estos sitios de inspección y resultados similares fueron encontrados por Souza y col (2007). En el presente estudio se observó una baja intensidad de parásitos en esófago y canal $(1,4 \%$ del total de casos) y no se encontraron casos en lengua, similar a lo registrado por Carvalho y col (2006) quienes encontraron en la canal $0,7 \%$ y en lengua $1,1 \%$. Sin embargo en Kenia, Wanzala y col $\left(2003^{\text {b }}\right)$ observaron una baja intensidad de infección en sitios de inspección oficial, encontrando cisticercos sólo en corazón (36\%), lengua (12\%), y braquial (16\%); en África también se incluye el músculo tríceps braquial (paleta) en la inspección oficial. En Etiopía, Abunna y col (2008) demostraron la misma tendencia: corazón $29,2 \%$ seguido por músculos maseteros $26,7 \%$, músculos de la paleta $25,3 \%$, lengua $10,4 \%$, diafragma $5,4 \%$ y $2,8 \%$ en otros sitios como el hígado, pulmón y riñón.

En Brasil, se observó cisticercos en corazón (11\%) y músculo maseteros $(8,5 \%)$ en bovinos infectados experimentalmente (Lopes y col 2011).

En el cuadro 1 se muestran los resultados de la inspección oficial y de la disección de los 33 cortes de carne oficiales examinados en las 20 medias canales, de las cuales sólo una correspondió a infección masiva (canal $\mathrm{n}^{\circ} 12$ ), siendo decomisada (MINSAL 2002). En la canal con infección masiva se encontraron 18 cisticercos en la

Cuadro 1. Distribución de cisticercos de Taenia saginata en los sitios de inspección oficial y en los cortes de carne de 20 canales de bovinos naturalmente infectados.

Distribution of cysticerci of Taenia saginata in the mandatory inspection sites and in the meat cuts of 20 naturally infected cattle carcasses.

\begin{tabular}{|c|c|c|c|c|c|c|c|c|c|c|c|c|c|c|c|c|c|c|c|c|c|c|}
\hline \multirow{2}{*}{$\begin{array}{l}\text { Sitios de } \\
\text { inspección }\end{array}$} & \multicolumn{20}{|c|}{ Número de la canal } & \multirow{2}{*}{$\begin{array}{c}\text { Total } \\
20\end{array}$} & \multirow[t]{2}{*}{$\%$ Tota } \\
\hline & 1 & 2 & 3 & 4 & 5 & 6 & 7 & 8 & 9 & 10 & 11 & 12 & 13 & 14 & 15 & 16 & 17 & 18 & 19 & 20 & & \\
\hline Corazón & 1 & 0 & 1 & 1 & 1 & 1 & 1 & 1 & 1 & 1 & 1 & 14 & 0 & 1 & 0 & 0 & 1 & 1 & 1 & 1 & 29 & 52,7 \\
\hline Maséteros & 0 & 1 & 0 & 0 & 0 & 0 & 0 & 0 & 0 & 2 & 0 & 3 & 1 & 0 & 1 & 1 & 1 & $1^{\mathrm{b}}$ & 0 & 0 & 11 & 20,0 \\
\hline Canal & 0 & 0 & 0 & 0 & 0 & 0 & 0 & 0 & 0 & 0 & 0 & $1^{\mathrm{a}}$ & 0 & 0 & 0 & 0 & 0 & 0 & 0 & 0 & 1 & 1,8 \\
\hline Total inspección & 1 & 1 & 1 & 1 & 1 & 1 & 1 & 1 & 1 & 3 & 1 & 18 & 1 & 1 & 1 & 1 & 2 & 2 & 1 & 1 & 41 & 74,6 \\
\hline Filete & 0 & 0 & 0 & 0 & 0 & 0 & 0 & 0 & 0 & 0 & 0 & 1 & 0 & 0 & 0 & 0 & 0 & 2 & 0 & 0 & 3 & 5,4 \\
\hline Tapapecho & 0 & 0 & 0 & 0 & 0 & 0 & 0 & 0 & 0 & 0 & 0 & 0 & 0 & 0 & 0 & 0 & 0 & 2 & 0 & 0 & 2 & 3,6 \\
\hline Asiento & 0 & 0 & 0 & 0 & 0 & 0 & 0 & 0 & 0 & 0 & 0 & 1 & 0 & 0 & 0 & 0 & 0 & 1 & 0 & 0 & 2 & 3,6 \\
\hline Ganso & 0 & 0 & 0 & 0 & 0 & 0 & 0 & 0 & 0 & 0 & 0 & 1 & 0 & 0 & 0 & 0 & 0 & 0 & 0 & 0 & 1 & 1,8 \\
\hline Plateada & 0 & 0 & 0 & 0 & 0 & 0 & 0 & 0 & 0 & 0 & 0 & 0 & 0 & 0 & 0 & 0 & 0 & 0 & 1 & 0 & 1 & 1,8 \\
\hline Asado de Tira & 0 & 0 & 0 & 0 & 0 & 0 & 0 & 0 & 0 & 0 & 0 & 1 & 0 & 0 & 0 & 0 & 0 & 0 & 0 & 0 & 1 & 1,8 \\
\hline Posta Negra & 0 & 0 & 0 & 0 & 0 & 0 & 0 & 0 & 0 & 0 & 0 & 0 & 0 & 0 & 0 & 0 & 0 & 1 & 0 & 0 & 1 & 1,8 \\
\hline Tapabarriga & 0 & 0 & 0 & 0 & 0 & 0 & 0 & 0 & 0 & 0 & 0 & 0 & 0 & 0 & 0 & 0 & 1 & 0 & 0 & 0 & 1 & 1,8 \\
\hline Posta de paleta & 0 & 0 & 0 & 0 & 0 & 0 & 0 & 0 & 0 & 0 & 0 & 0 & 0 & 0 & 0 & 0 & 1 & 0 & 0 & 0 & 1 & 1,8 \\
\hline Entraña & 0 & 0 & 0 & 0 & 0 & 0 & 0 & 0 & 0 & 0 & 0 & 1 & 0 & 0 & 0 & 0 & 0 & 0 & 0 & 0 & 1 & 1,8 \\
\hline Total cortes carne & 0 & 0 & 0 & 0 & 0 & 0 & 0 & 0 & 0 & 0 & 0 & 5 & 0 & 0 & 0 & 0 & 2 & 6 & 1 & 0 & 14 & 25,4 \\
\hline Total general & 1 & 1 & 1 & 1 & 1 & 1 & 1 & 1 & 1 & 3 & 1 & 23 & 1 & 1 & 1 & 1 & 4 & 8 & 2 & 1 & 55 & 100,0 \\
\hline
\end{tabular}

${ }^{a}$ Cisticerco encontrado a la inspección médico veterinaria en el miembro posterior.

${ }^{\mathrm{b}}$ Cisticerco viable diagnosticado en laboratorio. 
inspección oficial; adicionalmente se encontraron 5 cisticercos en total en los cortes de carne, los que estaban distribuidos en filete, asiento, ganso, asado de tira y entraña. En 16 (84,2\%) de las 19 canales con infección leve no se encontró ningún cisticerco en los cortes de carne a la disección, en tanto en las 3 canales con cortes parasitados, una tenía sólo un cisticerco en la plateada, otra tenía dos cisticercos en posta de paleta y tapabarriga respectivamente, y la tercera tenía 6 cisticercos repartidos en filete (2), tapapecho (2), asiento (1) y posta negra (1). En general, el total de cisticercos, así como su número por corte (máximo de 2), en las infecciones naturales resultaron muy inferiores a los datos de Lopes y col (2011) que mostraron una distribución mayor de los cisticercos en músculos esqueléticos $(75 \%)$ y en vísceras $(25 \%)$ en infecciones experimentales; resultando el hombro $(12,6 \%)$, cuello $(8,3 \%)$, lomo y filete $(7,3 \%)$, nudillo $(6,6 \%)$ y costillas $(5,5 \%)$ como sitios preferenciales. Minozzo y col (2002), en infecciones experimentales, observaron un $81,3 \%$ de los cisticercos en músculos de la parte delantera y trasera, mientras que, Wanzala y col $\left(2003^{\text {b }}\right)$, registraron una mayor frecuencia de cisticercos en vísceras $(22 \%$ en hígado y $17,1 \%$ corazón), seguido por los miembros posteriores $(15 \%)$ y anteriores $(13,4 \%)$ y pecho $(6,5 \%)$. Estos últimos autores, observaron que dicho patrón era revertido en infecciones naturales: miembros anteriores $(24,7 \%)$, miembros posteriores $(23,9 \%)$, pecho $(9,2 \%)$, hígado (12,2\%) y corazón $(8,8 \%)$. Igualmente, Almeida y col (2006) encontraron un alto nivel de parasitismo en el hígado 69,56\%. Las diferencias en la distribución de los cisticercos en los sitios de predilección, probablemente se deben a las diferentes zonas geográficas y sus prevalencias, así como a la actividad diaria y diferencia de edades de los animales beneficiados, la densidad de la población en la zona de origen de los animales beneficiados, sistemas de crianza y la cinética de la sangre, todos factores determinantes de la distribución anatómica de los cisticercos en el animal (Wanzala y col 2003 ${ }^{\text {, }}$ Carvalho y col 2006, Souza y col 2007, Lopes y col 2011).

El análisis macroscópico de los 148 casos de cisticercosis encontrados en la inspección médico veterinaria en el matadero, reveló un caso de cisticerco viable (integro) y se evidenció un aspecto cístico, con una pared translúcida y escólex en su interior. En los otros 147 casos los cisticercos estaban degenerados y poseían aspecto caseoso, caseoso calcáreo, diámetro en torno a $0,3 \mathrm{~cm}$, coloración blanquecina o amarillenta y consistencia firme. Por otra parte, el $88,0 \%$ fue confirmado positivo a cisticercos de Taenia saginata mediante histopatología, en tanto el $10,7 \%$ correspondió a otros diagnósticos, y en el 1,3\% de los casos no se encontró ninguna alteración al examen histopatológico. Entre los otros diagnósticos, se encontraron principalmente procesos inflamatorios como miocarditis, epicarditis, necrosis miocárdica, calcificación (miocárdica y del masétero) y miositis; alteraciones también reportadas por Silva y col (2011), lo que no descarta que podrían corresponder a una reacción a cisticercos en algún momento (Vera y col 2011), y actinobacilosis en lengua. Adicionalmente, del total de los casos de cisticercosis diagnosticados a la inspección oficial se observó el predominio de la condición de cisticercos degenerados con un $94,2 \%$ y sólo un $5,8 \%$ era viable. Solamente los cisticercos viables serían peligrosos como zoonosis (Ogunremi y Benjamin 2010). Por otra parte, sólo en una de las 20 canales diseccionadas totalmente el cisticerco encontrado en la inspección oficial fue diagnosticado como viable y fue precisamente en esa misma canal donde se hallaron más cisticercos en los cortes de carne, aunque todos éstos se encontraban degenerados (cuadro 1). Si bien de acuerdo a los resultados del presente estudio la inspección de la carne realizada según la Norma General Técnica No 62 de Chile (MINSAL 2002) parece adecuada desde el punto de vista de salud pública, se estaría sobrediagnosticando en un $12 \%$; desde este punto de vista el agregar un método complementario a la inspección visual para apoyarla, como podría ser la microscopía e histología, parece recomendable para reducir algunas pérdidas económicas innecesarias por saneamiento.

En las 14 lesiones compatibles con cisticercos de T. saginata en los cortes de carne, el estudio histopatológico confirmó la identificación, pero ninguno era viable (cuadro 1). Moreira y col (2002) encontraron un 6,4\% y Carvalho y col (2006) un 1,2\% de cisticercos viables en canal, respectivamente. En el presente estudio, el hallazgo en la canal de sólo cisticercos degenerados significa que éstos no tienen importancia en la transmisión de la infección a las personas, ya que para ello, se requiere el consumo de carne cruda o poco cocinada con cisticercos viables (Ogunremi y Benjamin 2010). Por otra parte todas las canales de animales con cisticercosis leve a la inspección son sometidas a saneamiento por congelación a $-20^{\circ} \mathrm{C}$ por 10 días (MINSAL 2002), proceso que inactivará cualquier cisticerco viable no identificado en la inspección.

En comparación con otros trabajos se observó una gran variabilidad en cuanto a los porcentajes de cisticercos degenerados y viables, aunque en general se ha demostrado un predominio de cisticercos degenerados (Santos y col 2003, Almeida y col 2006, Carvalho y col 2006, Souza y col 2007, Abunna y col 2008). Sin embargo, Wanzala y col $\left(2003^{\mathrm{b}}\right)$ demostraron una alta densidad de cisticercos viables y degenerados en diferentes sitios a la disección total en Kenia. En tanto que, Kebede (2008) registró un $85 \%$ y un $14,4 \%$ de cisticercos viables y degenerados, respectivamente en Etiopía. Como África se encuentra entre las regiones con más alta prevalencia de cisticercosis (Regassa y col 2009), es posible que esto influya en encontrar más cisticercos vivos. La re-estimulación de la inmunidad de los animales tras la invasión continua de oncósferas, explicaría el desarrollo de una fuerte inmunidad que no permite el desarrollo de más cisticercos y contribuye a explicar la ocurrencia tanto de cisticercos viables como degenerados en los sitios de 
predilección examinados en regiones de alta prevalencia (Wanzala y col 2003', Lopes y col 2011).

Considerando el total de cisticercos observados tanto a la inspección oficial (MINSAL 2002) como a la disección total de los cortes de carne de las 20 medias canales disecadas, el 74,55\% de los quistes fueron observados a la inspección oficial y sólo el $25,45 \%$ en cortes de carne. Esto confirma que los cisticercos parasitan preferentemente los sitios inspeccionados oficialmente, destacándose que en 16 de las 20 canales diseccionadas totalmente no se encontró ningún cisticerco adicional a los ya encontrados al examen oficial (cuadro 1). Esto coincide con un estudio experimental que demostró que los sitios inspeccionados para la detección de este parásito son tan buenos o mejor que cualquiera de los sitios no oficiales de inspección (Scandrett y col 2009). Por el contrario, en infecciones naturales y experimentales, Wanzala y col $\left(2003^{\mathrm{b}}\right)$, observaron cisticercos en todas las partes de las canales, diferentes a los sitios de inspección oficial, por lo cual para ese caso serían igualmente importantes como sitios de predilección de los cisticercos y podrían ser igualmente utilizados durante la inspección de rutina de la canal en los mataderos. Llama la atención que si bien hay estudios que demuestran que las vísceras verdes no son infectadas por cisticercos (Moreno 2003, Wanzala y col 2003 ), éstas igualmente son decomisadas en Chile (MINSAL 2002) cuando se encuentra algún cisticerco en los lugares de inspección oficial. Al respecto Moreno (2003) indica que la grasa y despojos blancos no precisarían el tratamiento de saneamiento, puesto que son utilizados previa cocción prolongada y no suelen presentar cisticercos. Considerando que las vísceras verdes son sometidas a un proceso térmico a altas temperaturas en Chile, ellas no constituirían un riesgo para la salud de la población y el decomiso actualmente ocasionaría pérdidas económicas innecesarias en la cadena de la carne.

En conclusión, la posibilidad de encontrar cisticercos en los cortes de carne de bovino es limitada, especialmente cuando la mayoría de los animales están infectados en forma leve, como sucede en el sur de Chile. Adicionalmente, la mayoría de los cisticercos encontrados en la inspección y disección total fueron no viables, de manera que no representan un alto riesgo para las personas consumidoras. Un programa de control ha de incluir la intervención en distintas etapas del ciclo de T. saginata, no siendo suficiente el diagnóstico en los mataderos. En especial se requiere poner énfasis en la educación general de las personas en cuanto a medidas higiénico-sanitarias para así evitar la diseminación de los huevos de $T$. saginata hacia los lugares donde los bovinos pastorean y beben agua. Finalmente, los resultados indican un sobre diagnóstico de cisticercosis de $12 \%$, lo que sugiere introducir una tecnología que permita complementar el examen visual en los mataderos, como por ejemplo el uso de microscopio estereoscópico o estudio histológico, para atenuar la pérdida económica por este concepto.

\section{AGRADECIMIENTOS}

Al proyecto INNOVA-CORFO 07CN13IAT247 por el financiamiento de este estudio y a la Agencia de Cooperación Internacional de Chile (AGCI) por financiar el programa de Magíster en Salud Animal de la primera autora.

\section{REFERENCIAS}

Abunna F, G Tilahun, B Megersa, A Regassa, B Kumsa. 2008. Bovine cysticercosis in cattle slaughtered at Awassa municipal abattoir, Ethiopia: prevalence, cyst viability, distribution and its public health implication. Zoon Public Health 55, 82-88.

Almeida DO, HP Igreja, FM Alves, IF Santos, R Tortelly. 2006. Cisticercose bovina em matadouro-frigorífico sob inspeção sanitária no município de Teixeira de Freitas-BA: Prevalência da enfermidade e análise anatomopatológica de diagnósticos sugestivos de cisticercose. Rev Bras Ci Vet 13, 178-182.

Carvalho LT, RR Falcão, IF Dos Santos, AL Teixeira. 2006. Prevalência de cisticercose em bovinos abatidos em matadouro-frigorífico sob inspeção federal em Minas Gerais. Rev Bras Ci Vet 13, 109-112.

Cayo R F. 2011. Cisticercosis bovina en el ganado faenado en el sur de Chile: Prevalencia, distribución y viabilidad de Cysticercus bovis en bovinos naturalmente infectados. Tesis de Magíster, Facultad de Ciencias Veterinarias, Universidad Austral de Chile, Valdivia, Chile.

Eichenberger RM, R Stephan, P Deplazes. 2011. Increased sensitivity for the diagnosis of Taenia saginata cisticercus infection by additional heart examination compared to the EU-approved routine meat inspection. Food Control 22, 989-992.

Fernandes JOM, WAS Buzetti. 2001. Prevalência de cisticercose bovina em animais abatidos em frigoríficos sob inspeção Federal, de Araçatuba, SP. Higiene Alimentar 15, 30-37.

Geerts S. 1990. Taenia saginata: een eeuwig probleem? Verh K Acad Geneesk Belg 52, 537-564.

Giesecke WH. 1997. Prevalence and economic implications of taeniasis/cysticercosis in South Africa. In: Cysticercosis. Report on a workshop held at the Onderstepoort Veterinary Institute. Onderstepoort South Africa, August 18-19.

INN, Instituto Nacional de Normalización. 1999. Cortes de carne de bovino. Norma Chilena Oficial NCh. 1596, Of. 99, Santiago, Chile.

Kebede N. 2008. Cysticercosis of slaughtered cattle in northwestern Ethiopia. Res Vet Sci 85, 522-526.

Kebede N, G Tilahun, A Hailu. 2009. Current status of bovine cysticercosis of slaughtered cattle in Addis Abeba Abattoir, Ethiopia. Trop Anim Health 41, 291-294.

Kyvsgaard NC, B Ilsøe, SA Henriksen, P Nansen. 1990. Distribution of Taenia saginata cysts in carcasses of experimentally infected calves and its significance for routine meat inspection. Res Vet Sci 49, 29-33.

Lopes W, TR Santos, VE Soares, JL Nunes, RP Mendonça, RC Lima, CA Sakamoto, GH Costa, VT Soccol, GP Oliveira, AJ Costa. 2011. Preferential infection sites of Cysticercus bovis in cattle experimentally infected with Taenia saginata eggs. Res Vet Sci 90, 84-88. 
Luna LG. 1968. Manual of Histologic Staining Methods of the Armed Forces Institute of Pathology. $3^{\text {rd }}$ ed. McGraw-Hill Book Company, New York, USA, Pp 12-35.

Maeda GE, NC Kyvsgaard, P Nansen, HO Bogh. 1996. Distribution of Taenia saginata cysts by muscle group in naturally infected cattle in Tanzania. Prev Vet Med 28, 81-89.

Martano G, M Voghera, G Serra. 2010. Epidemiological survey on bovine cisticercosis in Pinerolo District, Northwest Italy. Proceedings XXVI World Buiatrics Congress. Santiago, Chile, Pp 267.

Minozzo JC, RLF Gusso, EA de Castro, O Lago, VT Soccol. 2002. Experimental bovine infection with Taenia saginata eggs: recovery rates and cysticerci location. Braz Arch Biol Technol 45, 451- 455.

MINSAL, Ministerio de Salud. 2002. Norma General Técnica $\mathrm{N}^{\circ} 62$ sobre Inspección Médico Veterinaria de las reses de abasto y de sus carnes y criterios para la clasificación de aptitud para el consumo humano. MS, Santiago, Chile, Pp 1-64.

Moreira M, LP Almeida, DO Reis, WL Santos. 2002. Cisticercose bovina: um estudo com bovinos abatidos em matadouro municipal de Uberlândia, MG. Higiene Alimentar $16,37-41$

Moreno B. 2003. La Cisticercosis en la Inspección de Carnes. En: Moreno B (ed). Higiene e inspección de carnes. Vol II. Días de Santos, Madrid, España, Pp 268-277.

Ogunremi O, G MacDonald, S Geerts, J Brandt. 2004. Diagnosis of Taenia saginata cysticercosis by immunohistochemical test on formalin-fixed and paraffin-embedded bovine lesions. J Vet Diagn Invest 16, 438-441.

Ogunremi O, J Benjamin. 2010. Development and field evaluation of a new serological test for Taenia saginata cysticercosis. Vet Parasitol 169, 93-101.

OIE, Office Internationale des Epizooties. 2009. Chapter 2.4.4, Bovine Cysticercosis. In: OIE Manual of Diagnostic Tests and Vaccines for Terrestrial Animals. $5^{\text {th }}$ edition, Paris, France, Pp 1216-1226.

OPS/OMS, Organización Panamericana de la Salud y Organización Mundial de la Salud. 2003. Cisticercosis (CIE-10 B69). En: Acha PN, B Szyfres (eds). Zoonosis y enfermedades transmisibles comunes al hombre y los animales.
Vol 3. $3^{\text {a }}$ ed. OPS/OMS, Washington, Estados Unidos, Pp 171-181.

Regassa A, F Abunna, A Mulugeta, B Megeria. 2009. Major metacestodes in cattle slaughtered at Wolaita Soddo $\mathrm{Mu}-$ nicipal abattoir, Southern Ethiopia: Prevalence, cyst viability, organ distribution and socioeconomic implications. Trop Anim Health Prod 41, 1495-1502.

Santos R, IF Santos, JC Bonisson. 2003. Estudo comparativo entre a técnica post mortem de Santos e a do Serviço de Inspeção Estadual para a detecção do Cysticercus bovis em matadouros no estado do Rio de Janeiro, Brasil. Rev Bras Ci Vet, 10, 175-181.

Scandrett B, S Parker, L Forbes, A Gajadhar, P Dekumyoy, J Waikagul, D Haines. 2009. Distribution of Taenia saginata cysticerci in tissues of experimentally infected cattle. Vet Parasitol 164, 223-231.

Silva D, F Vera, M Moroni, E Paredes. 2011. Descripción histopatológica de lesiones compatibles con cisticercosis bovina. Resúmenes X Congreso Chileno de Buiatría, Osorno, Chile, Pp 135-136.

Souza V, MD Pessôa-Silva, M Kowalczuk, S Marty, V ThomazSoccol. 2007. Anatomic regions of major occurrence of Cysticercus bovis in bovines under federal inspection at slaughterhouse in the municipality of São José dos Pinhais, State of Paraná from July to December, 2000. Rev Bras Parasitol Vet 16, 92-96.

Vera F, D Silva, M Moroni, E Paredes. 2011. Schwannoma, diagnóstico histopatológico diferencial de cisticercosis bovina. Resúmenes X Congreso Chileno de Buiatría, Osorno, Chile, Pp 137-138.

Wanzala W, JA Onyango-Abuje, EK Kang'ethe, KH Zessin, NM Kyule, MPO Baumann, H Ochanda, LJS Harrison. $2003^{\text {a }}$. Analysis of post mortem diagnosis of bovine cysticercosis in Kenyan cattle. Online J Vet Res 1, 28-31.

Wanzala W, JA Onyango-Abuje, EK Kang, KH Zessin, NM Kyule, MPO Baumann, H Ochanda, LJS Harrison, 2003 . Control of Taenia saginata by post mortem examination of carcasses. Afr Health Sci 3, 68-76.

Yoder DR, ED Ebel, DD Hancock, BA Combs. 1994. Epidemiologic findings from an outbreak of cysticercosis in feedlot cattle. J Am Vet Med Assoc 205, 45-50. 